\section{Amorphous iron nanoparticles: special structural and physicochemical features enable chemical dynamic therapy for tumors}

\author{
"Recently, we have presented a novel hubble-bubble \\ approach to synthesize AFe-NPs, which show remarkable \\ advantages over the corresponding nanocrystalline \\ counterpart in serving as theranostic agents benefiting from \\ their amorphous nature."
}

First draft submitted: 2 February 2016; Accepted for publication: 10 March 2016; Published online: 15 April 2016

Keywords: amorphous metals • chemical dynamic therapy • iron nanoparticles

Amorphous metals or alloys, also known as the metallic glasses with a disordered structure on atomic level, have attracted great attentions owing to their special physical properties and extraordinary prosperities [15]. In addition to the excellent plasticity upon heating for easy processing, the absence of grain boundaries leads the bulk metallic glasses (BMGs) to higher strength and better corrosion resistance than conventional metals or alloys, which stimulated widespread researches to hurry their potential as advanced structural materials. However, the severe synthesis conditions, typically the superhigh cooling rate (usually higher than $10^{6} \mathrm{~K} \mathrm{~s}^{-1}$ ) to freeze the molten metal and to suppress the nucleation of crystalline phases, have been being one of the main bottlenecks for the widespread applications of BMGs [6].

Compared with BMGs, nanosized amorphous materials such as amorphous nanoparticles, which combine the advantages of both random arrangement of atoms in amorphous state and nanodimension and are therefore expected to exhibit even more unique and diverse properties, have been rarely explored, most probably due to the difficulties in the successful syntheses of such materials which leaves rooms for researches and developments.
Taking amorphous iron nanoparticles (AFe-NPs) as an example, not to mention the possible specificities in electromagnetism such as soft magnetic characteristics and others, it is expected that the randomly arranged iron atoms are active enough to be ionized in mild acidic conditions for on-demand $\mathrm{Fe}$ (II) release and to initiate subsequent localized Fenton reaction under the presence of hydrogen peroxide. On the other hand, most solid tumors feature acidic nature $(\mathrm{pH}$ = 6.5-6.9) and overproduced hydrogen peroxide, and such a special microenvironment has attracted great interest for the exploration of so-called microenvironment-responsive cancer therapy [7-11]. In fact, acidic microenvironment in tumor has triggered the development of numerous strategies of $\mathrm{pH}$ stimuli-responsible controlled drug releases, in which drug is expected to release in acidic tumor but not in neutral normal tissues or blood stream; while cumulative $\mathrm{H}_{2} \mathrm{O}_{2}$ in cancer has been exploited to implement specific therapeutic strategies, such as to act as a spontaneous trigger for responsive-drugreleasing chemotherapy or an endogenous $\mathrm{O}_{2}$ producer for enhanced photodynamic therapy [12-14]. Thus, seemly totally different fields of metallic glasses and cancer therapies find their common points for combination.

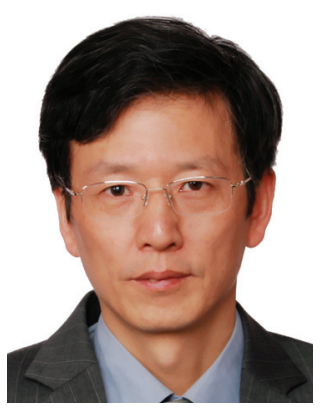

Jianlin Shi

State Key Laboratory of High Performance Ceramics \& Superfine Microstructure, Shanghai Institute of Ceramics, Chinese Academy of Sciences, Shanghai 200050, PR China jlshi@mail.sic.ac.cn 
Recently, we have presented a novel hubble-bubble approach to synthesize AFe-NPs, which show remarkable advantages over the corresponding nanocrystalline counterpart in serving as theranostic agents benefiting from their amorphous nature [15]. The successful synthesis is achieved by preventing the nucleation and growth of crystalline iron nanoparticles in an aqueous bubble film confined by the amphiphilic F-127 bilayers to suppress the long-range diffusion of the reduced $\mathrm{Fe}$ atoms. The unique advantages of such AFe-NPs have been evidenced for the theranostic applications in their report as follows. The amorphization of iron nanoparticles provide a potential but universal strategy to improve the biodegradability of inorganic biomedical agents such as drug carriers, MRI agents and so on, as the biodegradability of the inorganic agents has become one of the most concerned issues for their future clinical applications in comparison with the organic or polymeric ones, though these materials exhibit a number of attractive merits such as easy control of their dimensions and morphology, abundant strategies available for surface modification and multifunctionalization, high imaging performance or controlled drug release and so on; the amorphous state of iron nanoparticles show distinct magnetic properties, such as soft magnetism with a much lower coercivity $\left(\mathrm{H}_{\mathrm{c}}=59 \mathrm{Oe}\right.$ at $\left.300 \mathrm{~K}\right)$ and remanent magnetic induction $\left(B_{r}=12.1 \mathrm{emu}\right.$ $\mathrm{g}^{-1}$ at $300 \mathrm{~K}$ ) but higher saturation magnetism than its crystalline counterpart, the absence of magnetocrystalline anisotropy in representative crystalline magnets, much improved dispersibility in aqueous solutions featuring much smaller critical dimension for aggregation and more attractively, significantly enhanced longitudinal $\left(\mathrm{T}_{1}^{-1}\right)$ and transverse $\left(\mathrm{T}_{2}^{-1}\right)$ relaxation rates for better performances of magnetic resonance imaging. The most outstanding advantage of AFe-NPs is its stimuli-responsible $\mathrm{Fe}^{2+}$ release, that is, AFe-NPs' ionization, in the mild acidic microenvironment of tumors benefiting from the active nature of the atoms in disorder state, which enables a tumorspecific intratumoral in situ Fenton reaction using the endogenous hydrogen peroxide in tumor tissues. Such an intratumoral Fenton reaction results in the efficient $\mathrm{H}_{2} \mathrm{O}_{2}$ disproportionation for efficient $\bullet \mathrm{OH}$ generation which is one of the most toxic radicals to cells [16-18]. Based on this character of AFe-NPs which perfectly matches the microenvironmental features of tumors, a concept of chemical dynamic therapy has been proposed and demonstrated both in vitro and in vivo. Essentially different from the extensively explored photodynamic therapy which is highly dependent on the exogenous light irradiation of specific wavelength for ROS generation and therefore suffers from the limited penetration of light in tissues and possible UV irradiation-induced tissue damage, the demonstrated chemical dynamic therapy is initiated by the on-demand release of ferrous ions in acidic tumor tissues, relying on the intratumoral microenvironment, but totally independent of external stimulations. Specifically, normal tissues or cells will hardly initiate such Fenton reactions due to the absence or insignificance of hydrogen peroxide overproduction or acidic microenvironment therein.

"...the demonstrated chemical dynamic therapy is initiated by the on-demand release of ferrous ions in acidic tumor tissues, relying on the intratumoral microenvironment, but totally independent of external stimulations.

Outstanding advantages do not gloss over the presence of some drawbacks. For example, the scalable synthesis of AFe-NPs, or other amorphous metal nanoparticles, is still difficult based on the present hubble-bubble strategy, which necessitates more facile approaches of material preparation; the easy oxidation of AFe-NPs in air will make its storage in protective media a prerequisite and surface modifications, for example, surface PEGylation for enhanced biocompatibility and dispersity, and specific moiety grafting for targeted drug delivery, is not so easy as on mesoporous silica nanoparticles.

Nevertheless, the defects cannot obscure the virtues, the reported AFe-NPs of amorphous nature, whose specific soft magnetic properties and microenvironment responsibility will be highly attractive to the researchers in the cancer theranostics, as revealed in the author' report. More meaningfully, the concept of amorphous metal/alloy nanoparticles may offer a novel pathway to explore the unique structural features, electric, dielectric and magnetic properties, electronic and/or energy transfer characteristics and the resultant performances, which may enable a bright future for a variety of practical applications in, for example, electromagnetic devices, adsorptions and heterogeneous catalysis, etc.

\section{Financial \& competing interests disclosure}

The authors have no relevant affiliations or financial involvement with any organization or entity with a financial interest in or financial conflict with the subject matter or materials discussed in the manuscript. This includes employment, consultancies, honoraria, stock ownership or options, expert testimony, grants or patents received or pending, or royalties.

No writing assistance was utilized in the production of this manuscript. 


\section{References}

1 Klement W, Willens R. Non-crystalline structure in solidified gold-silicon alloys. Nature 187, 869-870 (1960).

2 Liu YH, Wang G, Wang RJ et al. Super plastic bulk metallic glasses at room temperature. Science 315, 1385-1388 (2007).

3 Demetriou MD, Launey ME, Garret G et al. A damagetolerant glass. Nat. Mater. 10, 123-128 (2011).

4 Chen M. A brief overview of bulk metallic glasses. NPG Asia Mater. 3, 82-90 (2011).

5 Wang JQ, Liu YH, Chem MW et al. Rapid degradation of azo dye by Fe-based metallic glass powder. Adv. Funct. Mater. 22, 2567-2570 (2012).

6 Wang JQ, Liu P, Chen N et al. The ultrastable kinetic behavior of an Au-based nanoglass. Acta Mater. 79, 30-36 (2014).

7 Muhammad F. Guo M, Qi W et al. pH-Triggered controlled drug release from mesoporous silica nanoparticles via intracelluar dissolution of $\mathrm{ZnO}$ nanolids. J. Am. Chem. Soc. 133, 8778-8781 (2011).

8 Van Sluis R, Bhujwalla ZM, Raghunand $\mathrm{N}$ et al. In vivo imaging of extracellular $\mathrm{pH}$ using $1 \mathrm{H}$ MRSI. Magn. Reson. Med. 41, 743-750 (1999).

9 Estrella V. Chen T, Lloyd M et al. Acidity generated by the tumor microenvironment drives local invasion. Cancer Res. 73, 1524-1535 (2013).

10 Zan M, Li J, Luo S et al. Dual pH-triggered multistage drug delivery systems based on host-guest interaction-associated polymeric nanogels. Chem. Commun. 50, 7824-7827 (2014).
11 Mura S, Nicolas J, Couvreur P. Stimuli-responsive nanocarriers for drug delivery. Nat. Mater. 12, 991-1003 (2013).

12 De Gracia Lux C, Joshi-Barr S, Nguyen T et al. Biocompatible polymeric nanoparticles degrade and release cargo in response to biologically relevant levels of hydrogen peroxide. J. Am. Chem. Soc. 134, 15758-15764 (2012).

13 Zhang Y, Yin Q, Yin L et al. Chain-shattering polymeric therapeutics with on-demand drug-release capability. Angew. Chem. Int. Edit. Engl. 52, 6435-6439 (2013).

14 Muhammad F, Wang A, Miao L et al. Synthesis of oxidant prone nanosilver to develop $\mathrm{H}_{2} \mathrm{O}_{2}$ responsive drug delivery system. Langmuir 31, 514-521 (2014)

15 Zhang C, Bu WB, Ni DL et al. Synthesis of iron nanometallic glasses and their application in cancer therapy by a localized fenton reaction. Angew. Chem. Int. Edit. Engl. 55, 2101-2106 (2016).

16 Fenton $\mathrm{H}$. Oxidation of tartaric acid in presence of iron. J. Chem. Soc., Trans. 65, 899-910 (1894).

17 Wlassoff WA, Albright CD, Sivashinski MS et al. Hydrogen peroxide overproduced in breast cancer cells can serve as an anticancer prodrug generating apoptosisstimulating hydroxyl radicals under the effect of tamoxifenferrocene conjugate. J. Pharm. Pharmacol. 59, 1549-1555, (2007)

18 Lee D, Khaya S. Velasquez-Castano JC et al. In vivo imaging of hydrogen peroxide with chemiluminescent nanoparticles. Nat. Mat. 6, 765-769 (2007). 